\title{
Aprendizaje basado en un proyecto de gamificación: vinculando la educación universitaria con la divulgación de la geomorfología de Chile
}

\author{
Delia Rodríguez-Oroz \\ Centro de Investigación en Sustentabilidady Gestión Estratégica de Recursos. Facultad de Ingeniería. \\ Universidad del Desarrollo. Avenida Plaza 680.Santiago.Chile.mdrodriguez@udd.cl \\ ORCID: https:/ / orcid.org/0000-0002-9859-485X
}

\author{
Roberto Gómez-Espina \\ Facultad de Ingeniería. Universidad Andres Bello. Autopista Concepción-Talcabuano 7100. Concepción. \\ Chile.roberto.gomez@unab.cl \\ ORCID: https:// orcid.org/0000-0002-5577-1692
}

\author{
María Jesús Bravo Pérez \\ Facultad de Ingeniería. Universidad Andres Bello. Autopista Concepción-Talcahuano 7100. Concepción. \\ Chile.mbravop@unab.cl \\ ORCID: https:// orcid.org/0000-0002-4027-0676
}

\author{
María Elena Truyol \\ Facultad de Ingeniería. Universidad Andres Bello. Antonio V aras 880. Santiago. Chile. \\ maria.truyol@unab.cl \\ ORCID: http:/ / orcid.org/0000-0003-1153-4967
}

[Recibido: 4 Junio 2018. Revisado: 11 Septiembre 2018. Aceptado: 10 Febrero 2019]

\begin{abstract}
Resumen: Con el objetivo de promover el conocimiento de la geomorfología a través de una herramienta utilizable por el conjunto de la sociedad, en el curso de geomorfología de la carrera de geología se incorporó el aprendizaje basado en proyectos (ABP) y la gamificación como metodologías de enseñanza-aprendizaje innovadoras. El proyecto se basó en la creación de un juego de mesa al que se le suma la incorporación de la gamificación al momento de utilizar el juego creado, el cual mostró ser una herramienta de aprendizaje que favorece la adquisición de conocimientos geomorfológicos, la capacidad de análisis, creatividad y motivación en los estudiantes. Debido a la sencillez tecnológica y accesibilidad económica del juego creado, se espera que la sociedad tenga la posibilidad de acercarse a la geología, adquiriendo un mayor conocimiento de su entorno natural.
\end{abstract}

Palabras clave: Juegos de mesa; Gamificación; Divulgación; Educación en geociencias; Aprendizaje basado en proyectos.

Learning based on a gamification project: connecting university education and Chilean geomorphology dissemination

Abstract: With the aim of promoting knowledge of geomorphology through a tool that can be used by society as
a whole, in a geomorphology course of geological degree project-based learning (PBL) and gamification were
incorporated as an innovative teaching-learning methodology. The implementation of the project was based on
the creation of a board which incorporated the gamification at the moment of using the created game which
proved to be a viable learning tool which contributes to the acquisition of geomorphological knowledge,
improves the analytical abilities, creativity and motivation in students. Due to the technological simplicity and
economic accessibility of the game created, it is expected that society has the possibility of approaching geology,
becoming in a better knowledge of its natural environment.

Keywords: Table games; Gamification; Popularization; Geoscience education; Project-based learning. 
Para citar este artículo: Rodríguez-Oroz D., Gómez-Espina R., Bravo Pérez M. J., Truyol M. E. (2019) Aprendizaje basado en un proyecto de gamificación: vinculando la educación universitaria con la divulgación de la geomorfología de Chile. Revista Eureka sobre Enseñanza y Divulgación de las Ciencias 16 (2). $2202 . \quad$ doi: 10.25267/Rev_Eureka_ensen_divulg_cienc.2019.v16.i2.2202

\section{Introducción}

A lo largo de los años las metodologías de aprendizaje activo se han ido diversificando y mejorando progresivamente. A pesar de esto, el método de enseñanza predominante en geología sigue siendo el de transmisión en el aula (Tishman, Jay y Perkins 1993). La enseñanza de la geología ha estado ajena a los cambios y son pocas las instancias conocidas donde se han aplicado de manera sistemática diversas metodologías de enseñanza aprendizaje diferentes a la convencional (Gates y Kalczynski 2016). Un complemento a las clases expositivas han sido las salidas a terreno que, por lo demás, son una metodología tradicional dentro de las geo ciencias y tienen su acción principalmente dentro del ámbito universitario. El traslado de esta metodología de enseñanza a la sociedad se produce principalmente en campamentos de verano y rutas de interpretación en parques naturales (Davis 2002), pero su rango de alcance sigue siendo pequeño. Por tanto, la situación en la que nos encontramos muestra que la educación en geología se produce principalmente en las universidades y requiere de la adopción de un rol social tanto de los profesores como de los estudiantes de geología. Sin embargo, la verdadera divulgación y traspaso de conocimiento sólo se produce cuando es la sociedad la que participa de las actividades de enseñanza y aprendizaje. Por ello, para divulgar la geología y aumentar el conocimiento y entendimiento del entorno geológico que nos rodea es necesario tener una mayor audiencia fuera de las universidades. Es por esto que se hace necesario formar geólogos con habilidades en divulgación mediante el empleo de métodos distintos de los tradicionales, los cuales permitan facilitar la llegada de la geología a un mayor espectro social.

En Chile, un país en el que la minería ha sido y es el motor de la economía, existe una gran despreocupación por parte de la comunidad geológica en su labor de educador social. Hay un gran desconocimiento entre la población sobre los procesos naturales y el entorno geomorfológico, lo que hace que no se valore el rico y diverso patrimonio geológico que alberga el país. Chile ha realizado en los últimos 50 años un gran esfuerzo por aumentar el proceso de regionalización y descentralización y alcanzar un desarrollo más armónico que genere una mayor integración social y territorial (Gaete Feres 2006). Sin embargo, la verdadera integración pasa por que la población sea capaz de conocer, comprender, valorar y preservar el legado de los pueblos originarios y el entorno natural que es parte del legado cultural. Para ello, Chile necesita divulgadores de la geología que deben ser formados en las universidades a través del desarrollo de asignaturas, postgrados y títulos propios que entreguen herramientas y estrategias de divulgación científica que apunten a la apropiación social del conocimiento geo científico, labor esencial para lograr un verdadero desarrollo sustentable en el siglo XxI.

En este sentido, nuestra premisa es que la gamificación puede ser una herramienta de aprendizaje universal, lo que evidencian las múltiples incorporaciones de juegos en la enseñanza superior y la divulgación (Arambarri, Armentia y Baeza 2012, Iosup y Epema 2014, Urh, Vukovic y Jereb 2015, Gates y Kalczynski 2016). El entorno de juego favorece la motivación de los estudiantes y usuarios por aprender y ayuda a mejorar el entendimiento de conceptos complejos que requieren pensamiento espacio temporal. Las universidades, por sus especiales características, deben hacer un esfuerzo a la hora de proveer de nuevas metodologías y actividades de $\mathrm{I}+\mathrm{D}+\mathrm{i}$, bien sea por rescatar su semi olvidada función de divulgación del conocimiento o por la necesidad de adaptarse a los nuevos medios y usuarios (González Tardon 2015). Teniendo esto como base y buscando mejorar tanto la metodología 
ocupada como los resultados obtenidos, se planteó la aplicación de una actividad utilizando el Aprendizaje Basado en Proyectos (ABP) y, posteriormente, la gamificación, en un curso de geomorfología de $3^{\circ}$ año de la carrera de Geología. La estrategia de gamificación utilizada consistió en la creación de un juego de mesa que incorporó los conceptos adquiridos en clases dentro del contexto geomorfológico y patrimonial chileno, para luego ser ofrecido como una herramienta de aprendizaje y divulgación de la geología en colegios, centros de interpretación y hogares. Finalmente, el juego creado debía ser lo suficientemente sencillo y accesible como para ser usado fuera del ámbito universitario, poniendo la geología al alcance de la población a muy bajo costo.

\section{Marco teórico}

Tradicionalmente en Chile, debido a que los recursos minerales son uno de los principales motores de la economía, las mallas curriculares de las carreras de geología están más enfocadas en las asignaturas relacionadas con la geología económica. Sin embargo, existe una creciente demanda de geólogos desde las instituciones educativas, empresas de ingeniería y consulting o del sector turístico, lo cual exige una mayor versatilidad de estos profesionales. Resulta claro que, dada la inexistencia de asignaturas específicas que enseñen habilidades académicas para la divulgación científica en las mallas de las carreras de geología, existe la necesidad de enseñar a divulgar en las aulas, promoviendo métodos innovadores en la educación en geología que motiven a los alumnos y que deriven en el desarrollo de sus habilidades comunicativas y de divulgación. Es por esto que, mediante el diseño por parte de los alumnos del juego "Juego de Dominios Geomorfológicos", cuya creación se visualizó como una herramienta de ABP, se pretendió entregar un producto final a la comunidad que favorezca el aprendizaje basado en la gamificación, balanceando de este modo la brecha que existe entre los perfiles de egreso de los alumnos de geología, la realidad del mercado laboral y el escaso o nulo conocimiento de la geomorfología entre la sociedad.

El ABP es una estrategia pedagógica integral (Maldonado 2008), que se basa en el aprendizaje colaborativo y pretende que el estudiante logre resolver un problema a través del diseño y la implementación de un proyecto que integre distintas áreas del conocimiento. Centra su desarrollo en competencias técnicas como la investigación, análisis y experimentación, pero además fortalece competencias suaves como trabajo cooperativo, comunicación oral y generación de conocimiento entre otras aptitudes que son requeridas en un ambiente profesional (Williams 2014). En el ABP, el estudiante es el principal responsable de su proceso de aprendizaje en el que el docente tiene un rol de guía y facilitador de recursos (Cascales, Carrillo y Redondo 2017). La metodología ABP consta de una serie de fases: Inicio, primeras actividades de los equipos, desarrollo del proyecto, conclusiones desde la perspectiva del alumno y conclusiones desde la perspectiva del profesor (Gómez y Santos 2012). Existen numerosas experiencias del uso de ABP en cursos universitarios de ingeniería (ArellanoPimentel, Nieva-García y Algredo-Badillo 2013, Sánchez-Martínez, Rojas-Molina y SánchezFlores 2015), física (Holubova 2008) y geología (Smith, Hoersch y Gordon 1995). Sin embargo, en estos casos los proyectos buscan solucionar problemáticas intrínsecas a su disciplina dentro del marco del aprendizaje de los estudiantes. En este trabajo, además se pretende facilitar una herramienta que ayude a mejorar el desconocimiento existente en la sociedad acerca de las geo ciencias. Bell (2010) destaca que los ABP promueven el aprendizaje social de los estudiantes, haciéndolos más competentes con las habilidades de comunicación del siglo XXI.

La gamificación se describe como el proceso de pensamiento de juego y sus mecanismos para atraer a los usuarios y hacerlos resolver problemas (Zichermann y Cunningham 2011). En este sentido, la gamificación rescata la mecánica de los juegos y la pone a favor del aprendizaje 
(Marín-Díaz 2015) propiciando un ambiente de competencia y la motivación por aprender por parte de los estudiantes (Faiella y Ricciardi 2015) y usuarios en general, otorgando un feedback inmediato (Kapp 2012). Sin embargo, la literatura muestra una escasa implementación de la gamificación en las aulas. Tan solo el 11,3 \% de los profesores utilizan la gamificación como método de enseñanza (Martí-Parreño, Seguí-Mas y Seguí-Mas 2016), siendo especialmente escasos los trabajos enfocados a la geología (Morales 2012, González et al. 2012, Shipley, Tikoff, Ormand y Manduca 2013, Gates y Kalczynski 2016). Sin embargo, la relevancia de la gamificación en otras áreas de la educación universitaria se ha incrementado significativamente desde el 2012 (Yang, Chien y Liu 2012, De Bie y Lipman 2012, Lim y Ong 2012, Arambarri et al. 2012, Hamari, Koivisto y Sarsa 2014, González Tardon 2015). Otra de las ventajas de la gamificación es que permite que el aprendizaje funcione según las necesidades individuales de los alumnos en base al método ensayo-error y durante todo el proceso de aprendizaje (Hanus y Fox 2015). Sin embargo, se debe tener cuidado al aplicar la gamificación en los entornos educativos, pues existen estudios que muestran peores resultados en las evaluaciones (Hanus y Fox 2015) o disminución del disfrute y utilidad de la gamificación con el tiempo de uso (Koivisto y Hamari 2014).

\section{Objetivo de la innovación}

El artículo tiene dos objetivos relacionados con la implementación de innovación educativa en el aula a través de la creación del juego (Aprendizaje Basado en Proyectos) y el uso de éste como herramienta de divulgación de la geomorfología para la sociedad (Gamificación). En este contexto, el primer objetivo del proyecto es el desarrollo y construcción de un juego como herramienta pedagógica que contribuya a la mejora del progreso académico y facilite la adquisición del conocimiento geomorfológico a los alumnos de tercer curso de geología. El segundo es proporcionarles formación en metodologías de divulgación y comunicación principalmente en aprender a enseñar y trascender en su proceso formativo en un esfuerzo por aumentar la diversidad de conocimientos y las posibilidades laborales de nuestros egresados.

Además, a través de la prueba de la mecánica del juego en el aula se logra perfeccionar el producto final, mejorar los niveles de disfrute e interés a través de la automotivación de los alumnos.

Más allá de estos objetivos académicos del curso de geomorfología, se busca proporcionar una herramienta que permita la divulgación de ésta a través de la gamificación. Para ello se entrega el juego de mesa diseñado por los estudiantes, que permite a sus usuarios interpretar a través de las diferentes geoformas observadas sobre la superficie terrestre los procesos geomorfológicos que han esculpido paisaje y forman parte del patrimonio geo cultural chileno.

\section{Elementos del juego}

El juego se compone de dos elementos principales: información geomorfológica recogida en "tarjetas" y el soporte físico del juego "tablero". La información geomorfológica se entregó a los alumnos durante las clases teóricas y ésta se representó en las diferentes tarjetas y el tablero de juego diseñados por los alumnos en base al formato entregado por la profesora, de tal manera que permitieran a los estudiantes relacionar conceptos teóricos básicos de geomorfología, procesos y factores geológicos, con las geoformas del relieve características de los diferentes dominios climáticos. Las diferentes tarjetas contienen la siguiente información:

- Tarjetas de geoformas $(6 \mathrm{~cm}$ de ancho $\times 8 \mathrm{~cm}$ de alto): En estas tarjetas se muestran las geoformas que los alumnos consideraron más características de los diferentes dominios 
geomorfológicos identificados en Chile (ejemplo: terraza, morrena o cárcava), que contienen información relacionada con los procesos y factores geológicos que las originan. En el anverso muestran una imagen real y el nombre de una geoforma (figura 1 izquierda) y en el reverso indican el proceso y factor que las origina (figura 1 derecha). Estas tarjetas se diseñan en 5 colores asignando a cada color un dominio climático. El color blanco es representante del dominio glaciar, el verde del valle central, el azul marino del litoral, el azul celeste del fluvial, el amarillo del desértico y el rojo del volcánico. Además, dada la riqueza cultural de los pueblos originarios de Chile y su distribución a los largo del país se pudo identificar cada dominio geomorfológico con el territorio ocupado por estos pueblos. De esta forma, los distintos dominios geomorfológicos se identifican como pueblos nativos que los ocuparon. Los alacalufes se identifican con el dominio glaciar, los mapuches con el fluvial, los atacameños con el desértico, los puelches con el volcánico y los changos con el litoral.

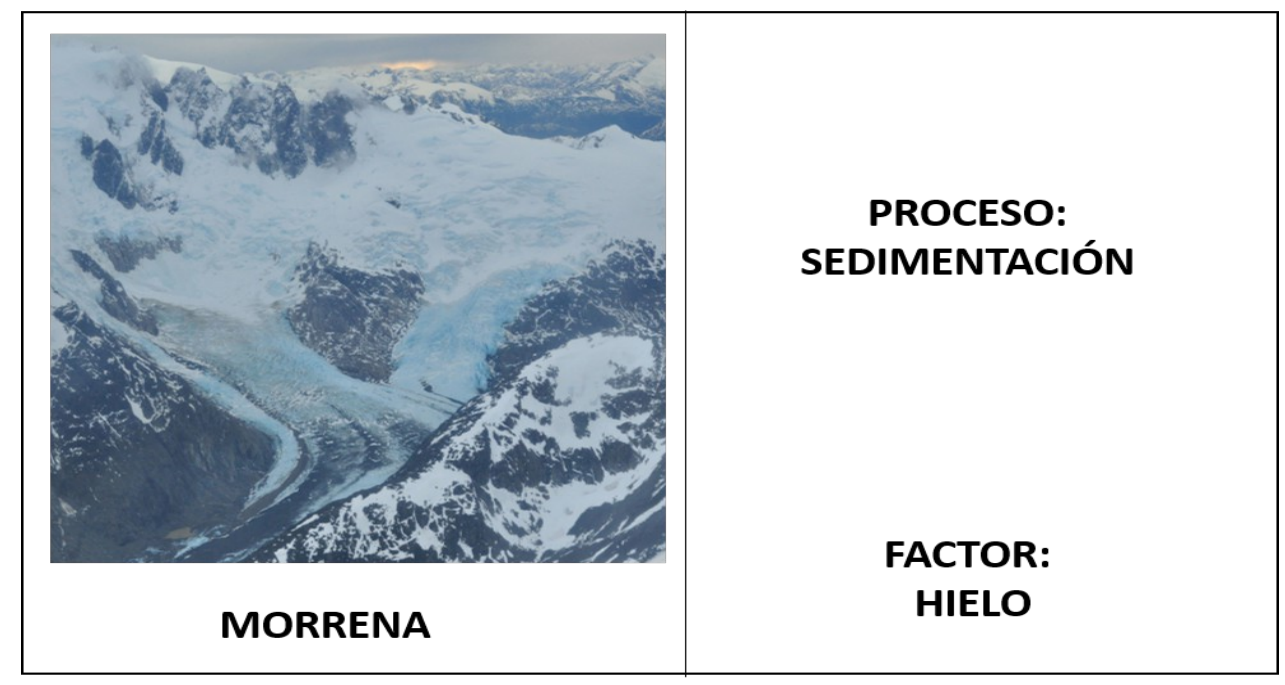

Figura 1. Ejemplo de tarjeta perteneciente al dominio glaciar (blanco) donde se muestra la geoforma de morrena originada por el proceso y factor correspondientes, sedimentación y hielo.

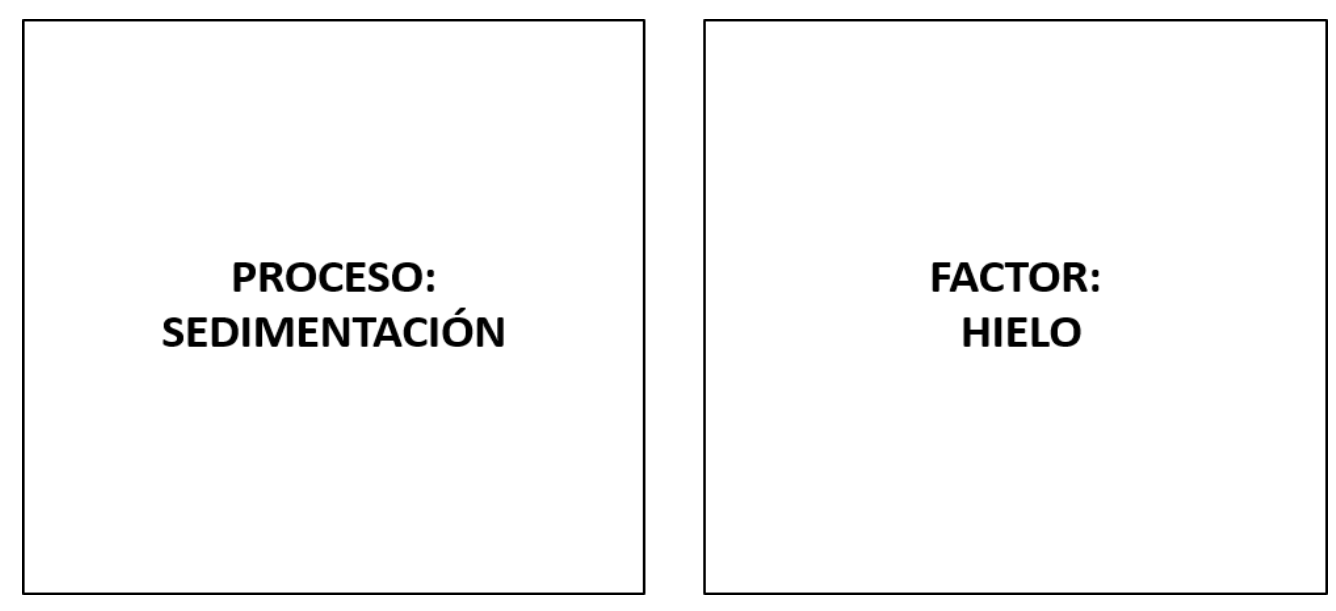

Figura 2. Ejemplo de tarjetas de recursos perteneciente al dominio glaciar (blanco) donde se muestra el proceso de sedimentación (izquierda) y el factor hielo (derecha).

- Tarjetas de recursos $(4 \mathrm{~cm}$ de ancho $\times 5 \mathrm{~cm}$ de alto): estas tarjetas son de dos tipos, unas contienen el nombre de los procesos (ejemplo: eustasia, glaciación, erosión, sedimentación) y las otras los factores (ejemplo: litología, pendiente o precipitación) que originan las geoformas. Las tarjetas tendrán el color que corresponda al dominio climático con el que se relacionan (figura 2). 
El segundo elemento del juego es el tablero (cartulina tamaño A4; $44 \mathrm{~cm}$ de largo $\times 28 \mathrm{~cm}$ de ancho) que representa el mapa de Chile, el cual se segmenta en diferentes sectores según los 5 dominios geomorfológicos más representativos que los alumnos identifican en el territorio chileno. Cada uno de los dominios geomorfológicos del mapa se identifica con un color en el tablero (figura 3). A su vez, aparecen simbolizados una serie de caminos que unen los dominios y que están divididos en casillas que pueden contener elementos trampa o sorpresa. Ubicado fuera del mapa existe un rectángulo de $4 \mathrm{~cm} \times 5 \mathrm{~cm}$ donde se colocan las tarjetas de los recursos (procesos y factores).

Algunos elementos del juego, como el tablero y las instrucciones del mismo, se pueden solicitar de forma gratuita al correo de contacto del autor principal o descargarse a través del enlace https://drive.google.com/drive/folders/1mudUHoNPiU6_asIRI1YXvZCRClvOs_-F? usp $=$ sharing. Sin embargo, las tarjetas de las geoformas que contienen imágenes no podrán entregarse pues los autores de las fotografías ostentan los derechos de autor de las mismas.

\section{Proceso de implementación}

La implementación se llevó a cabo en el curso de Geomorfología del $3^{\circ}$ año de la carrera de Geología de una Universidad chilena, en el semestre agosto-diciembre 2016. En la figura 4 se indica la caracterización de los integrantes del curso.

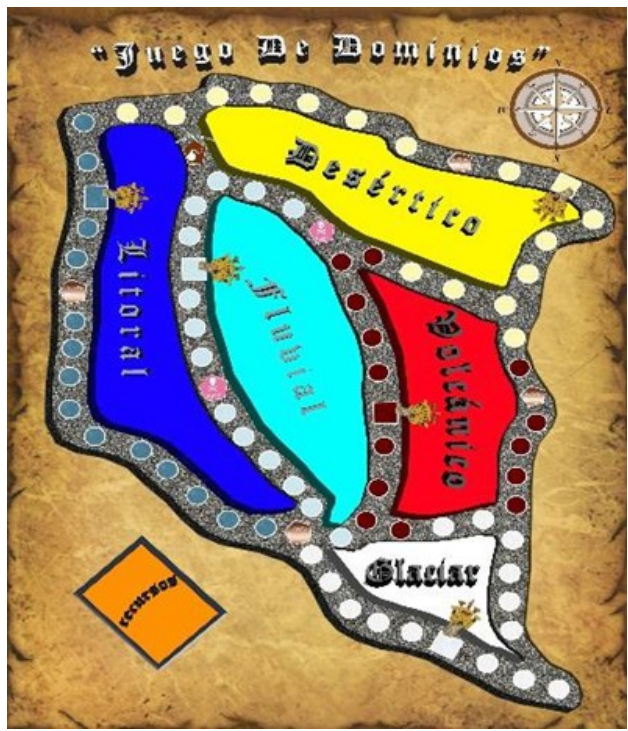

Figura 3. Diseño de tablero de juego.

Durante todo el proceso de creación del juego los estudiantes analizaron y estudiaron temas específicos para construir y definir los principales dominios geomorfológicos de Chile, sus geoformas más representativas y los procesos y factores que las generan. Este trabajo se realizó tanto en equipo como de forma individual, permitiendo a los alumnos adquirir de forma amena, significativa y progresiva los contenidos de la asignatura. Esta dinámica también permitió desarrollar la cooperación, la competencia, el sentimiento de logro y la identificación o arraigo por su entorno natural y los pueblos nativos. La dinámica propuesta para el juego fue muy parecida a los juegos de conquista, donde un grupo de estudiantes, representado por uno de los pueblos nativos chilenos, parte del dominio geomorfológico al que pertenece y se van desplazando por las casillas del tablero a la conquista de otros pueblos. Esta conquista de nuevos dominios geomorfológicos se realiza a través de la adquisición de sus geoformas representativas. Para ello el pueblo conquistador deberá tener las tarjetas de los procesos y factores que la definen y adquirirla.

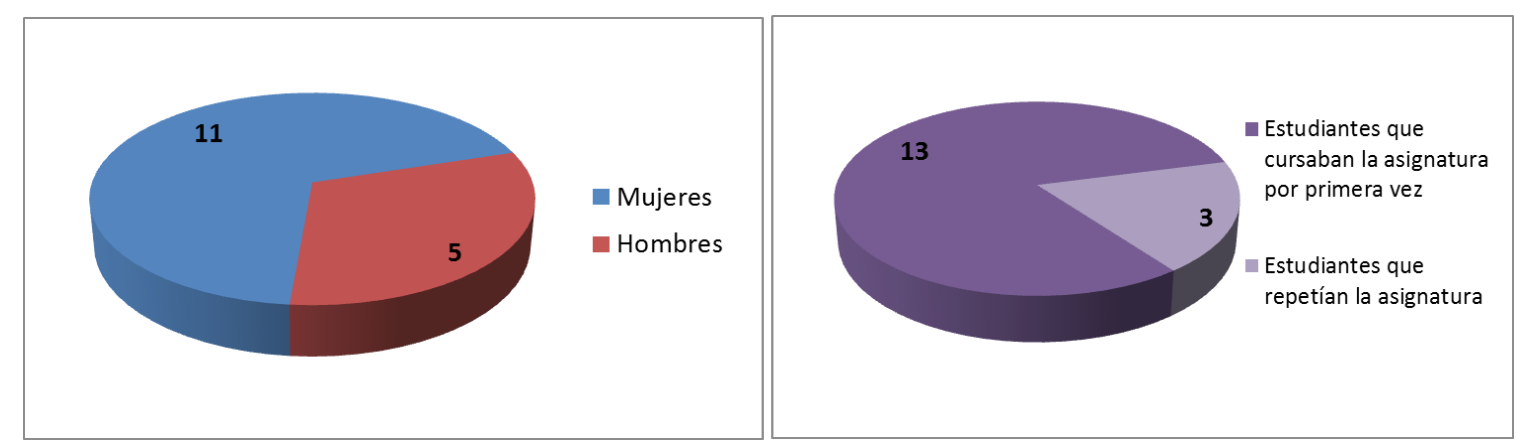

Figura 4. Caracterización de los estudiantes del curso GEO308 Geomorfología de 2016. 
Fue importante, previo a la creación del juego, clarificar los objetivos, reglas, derechos y responsabilidades de la profesora (primer autor) y de los integrantes de cada equipo. En primer lugar, la profesora fue la encargada de proporcionar estas bases y las fuentes de conocimiento específico, entregando el material de la asignatura "geomorfología", mientras que los estudiantes analizaron los temas, enfocándose en la interpretación de las geoformas y la caracterización geomorfológica del territorio chileno. De esta forma el trabajo puede dividirse en dos componentes:

\section{1) Creación del juego}

La creación del juego fue realizándose una vez por semana durante todo el semestre, permitiendo la interacción entre los conocimientos impartidos en clase, la participación grupal y la motivación al autoestudio. Esta actividad permitió a los estudiantes trabajar en los contenidos, profundizando en ellos para luego seleccionar los de mayor relevancia a ser incluido en el juego. En esta etapa de diseño participaron tanto la profesora como los alumnos. Si bien la libertad total de los alumnos podría haber generado una mayor creatividad, se consideró que ésta no tenía por qué ser necesariamente positiva para el desarrollo del juego. Por tanto, las bases del diseño del juego (dimensiones y componentes de las tarjetas y tablero) y las reglas fueron propuestas por la profesora, mientras que la elección de los dominios y sus características fueron seleccionadas por los alumnos a través de la aplicación de los contenidos vistos en las clases. La profesora creó 5 grupos de entre 3 y 4 alumnos, en los que distribuyó a los estudiantes de forma equitativa según el desempeño académico hasta la fecha y la retroalimentación a preguntas realizadas en clase. Los alumnos definieron los 5 dominios geomorfológicos más representativos de Chile $\mathrm{y}$, de forma consensuada, cada equipo seleccionó con qué dominio trabajar, concretando las geoformas más características del mismo e identificando para cada una los factores y procesos formadores. Estos factores y procesos serán los "recursos" necesarios para conquistar geoformas de otros dominios durante la etapa de juego y desarrollo de la innovación.

\section{2) Etapa de juego y desarrollo de la innovación}

Cada grupo de alumnos participó representando a un dominio geomorfológico a través de la figura de un pueblo originario chileno. Al inicio del juego cada pueblo tenía en su poder todas las tarjetas de geoformas de su dominio y su misión consistió en tratar de acumular recursos suficientes y específicos para poder conquistar las geoformas de los otros dominios. La figura 5 muestra la forma en cómo los alumnos se interrelacionaron durante el desarrollo del juego. Finalmente, a través de la adquisición del total de las geoformas de un dominio geomorfológico, cada pueblo podrá conquistar nuevos territorios.

En cada turno un equipo tira el dado, avanzando en el tablero el número de casillas obtenido en el mismo y recibiendo 3 tarjetas de recursos del mazo, entre las cuales habrá mezcladas cartas con poderes y con trampas, que aportan azar e intriga al desarrollo del juego. Cuando por avance un equipo caiga en la casilla de entrada de un dominio, podrá comprar una geoforma del mismo entregando los recursos necesarios. Durante el juego los equipos deben mostrar las geoformas de su dominio para que el resto pueda evaluar la conquista. La posibilidad de realizar trueques de recursos, la presencia de elementos sorpresa (ej.: terremotos, virus anta, etc.) o el pago de tributos a otros equipos, exige una estrategia grupal para que la conquista sea exitosa. Alcanzar la victoria en el juego radica en identificar correctamente la geoforma a conquistar con los recursos necesarios para ello. Al final el equipo que conquiste más dominios será el equipo con mayor productividad de conocimientos y mejor estrategia de equipo. 


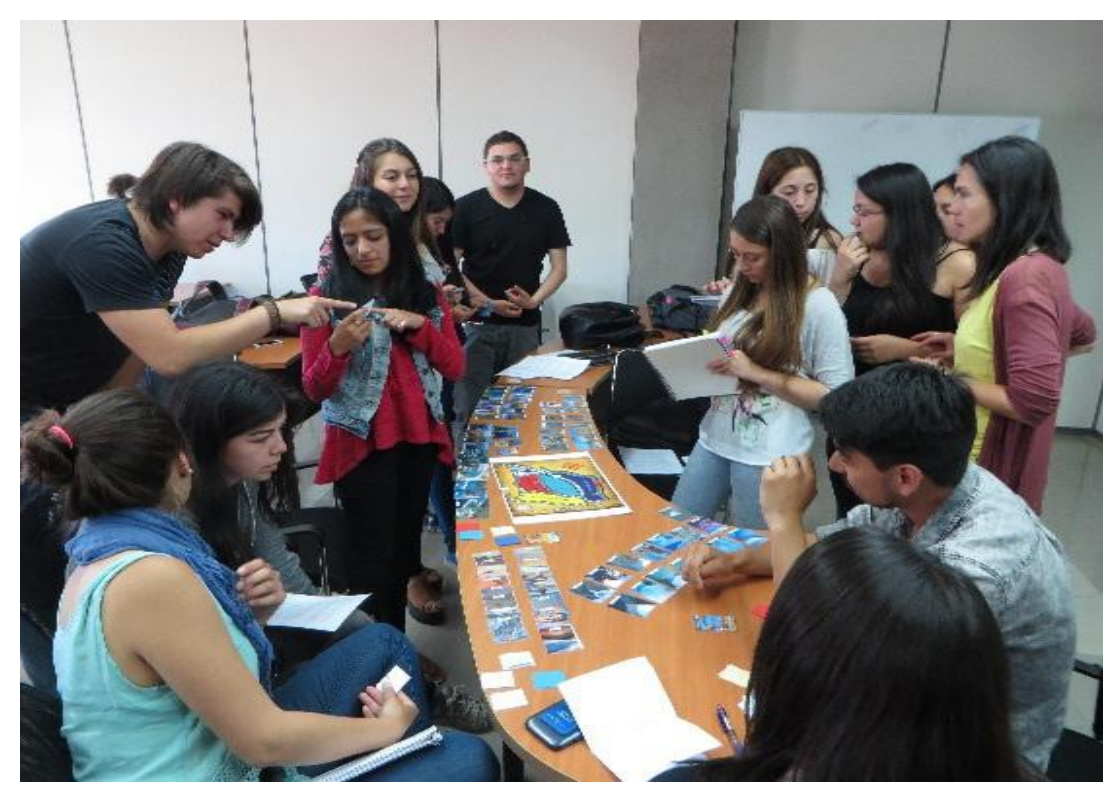

Figura 5. Sesión de juegos durante el curso.

\section{Metodología de recopilación y análisis de datos}

Para evaluar los contenidos vistos en la asignatura de geomorfología y trabajados durante el todo el semestre en la creación del juego se llevaron a cabo dos pruebas parciales (P1 y P2) durante el curso, un examen final (Ex), en el que se evaluó toda la materia, y una salida a terreno $(\mathrm{T})$, donde se aplicaron los conocimientos vistos en el aula. La nota final (NF) se obtuvo mediante la ponderación con las pruebas parciales, la final y el terreno según la siguiente fórmula: $\mathrm{NF}=\mathrm{P} 1 \times 0,3+\mathrm{P} 2 \times 0,3+\mathrm{Ex} \times 0,3+\mathrm{T} \times 0,1$. A efectos de interpretación de los datos cabe mencionar que en el sistema evaluativo del país en el que se encuadra este estudio (Chile), la escala de notas está comprendida entre 1,0 y 7,0, siendo la nota mínima de aprobación un 4,0.

El grado de satisfacción con la estrategia de la gamificación fue evaluado a través de una encuesta online anónima realizada en la web www.surveymonkey.com. Se facilitó el enlace web y se recolectaron las respuestas durante el período de clase y se analizaron más tarde. Todas las preguntas estaban en formato de opción múltiple y usaban una escala Likert. Las preguntas de la encuesta básica se diseñaron para determinar la visión general de la innovación y el interés en la geomorfología. En particular, las preguntas apuntaban a determinar en los estudiantes (1) comprensión general del contenido de geomorfología; (2) adecuación en la implementación de la innovación; (3) desarrollo de otras habilidades. Por lo tanto, las encuestas de referencia incluyeron muchas preguntas que no tenían relación con la geomorfología. Para obtener una valoración cuantitativa de estos aspectos se valorizaron las respuestas múltiples según estas hacían referencia a aspectos positivos o negativos de la innovación. Así, las preguntas referentes a aspectos positivos se valoraron con un 7,0 cuando el alumno estaba muy de acuerdo con la pregunta, 5,5 cuando estaba de acuerdo, 4,0 en caso de que su postura fuera neutral, 2,5 si estaba en desacuerdo con la pregunta y un 1,0 si se mostraba muy en desacuerdo. Para las preguntas referentes a aspecto negativos se empleó el criterio opuesto. 


\section{Resultados y análisis}

A continuación, se muestran y evalúan los resultados de las calificaciones obtenidas en cada año que se ha dictado la asignatura de geomorfología (figura 6). Debe señalarse que el año en el que se implementó la metodología ABP y de gamificación fue el 2016. Como se observa en la figura 6, los porcentajes de aprobación para 2016 mejoraron respecto a 2015 en 13,8 puntos porcentuales y respecto al 2014 en 26,6 puntos porcentuales, pudiendo confirmarse una mejora continua para el trienio 2014-2016 con independencia de la implementación de la metodología aplicada en el proceso de aprendizaje. Esto hace que no podamos concluir que exclusivamente la creación del juego y su uso hayan contribuido a la mejora de las tasas de aprobación.

A pesar de este aumento en el porcentaje de aprobación del curso, no se han observado variaciones significativas en cuanto al promedio de notas con la aplicación de la metodología activa (figura 6). Esto podría indicar que el conocimiento se ha adquirido de forma más homogénea entre el grupo de estudiantes, ya que la nota media se ha mantenido debido a que los puntajes más altos de aquellos alumnos destacados se equiparan con los del resto de la clase, lo que se cree es favorecido por el trabajo en grupos. Al igual que observó Mayer (2004), a lo largo de las actividades de preparación del juego se pudo observar que el trabajo en grupos favoreció el ambiente de aprendizaje que se tornó más dinámico, dando lugar a un auténtico aprendizaje activo, donde los alumnos de forma colaborativa analizaron las geoformas, las relacionaron con procesos geológicos y tradujeron sus ideas a un lenguaje simbólico, para concluir en una identificación de los dominios geomorfológicos de Chile accesible para todo tipo de público.

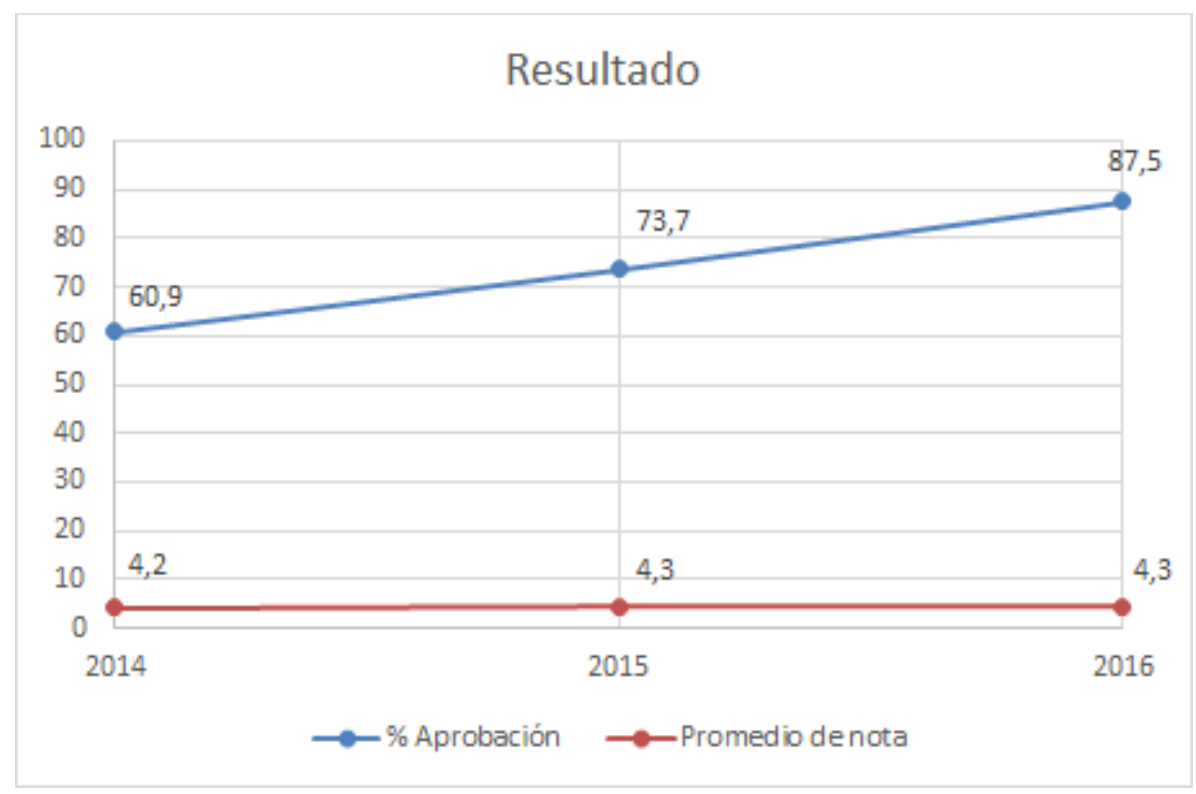

Figura 6. Evolución estadística anual entre los años 2014, 2015 y 2016 (23, 19 y 16 alumnos respectivamente) de los porcentajes de aprobación y promedio de nota de la asignatura Geomorfología (calificación máxima: 7,0).

Al finalizar la experiencia se pidió a cada alumno que indicara su grado de satisfacción con el desarrollo del proyecto a través de una encuesta de percepción online anónima. Se facilitó el enlace web y se recolectaron las respuestas durante el período de clase y se analizaron más tarde. Todas las preguntas estaban en formato de opción múltiple y usaban una escala Likert. Las preguntas de la encuesta básica se diseñaron para determinar la visión general de la 
estrategia de innovación y el interés en la geomorfología. En particular, las preguntas apuntaban a determinar en los estudiantes (1) comprensión general del contenido de geomorfología; (2) adecuación en la implementación de la innovación; (3) desarrollo de otras habilidades. Por lo tanto, las encuestas de referencia incluyeron muchas preguntas que no tenían relación con la geomorfología.

La figura 7 resume las valoraciones promedio que otorgaron los estudiantes en cada una de las preguntas. En relación con la opinión de los estudiantes, los indicadores definen una tendencia clara. Éstos muestran un alto grado de satisfacción frente a la dinámica ABP. En una escala del 1,0 al 7,0, el $60 \%$ de los encuestados valora la actividad con un 7,0 mientras que el $40 \%$ lo hace con un 5,6 , situando sus valoraciones en un alto grado de satisfacción con un puntaje de 6,4 .

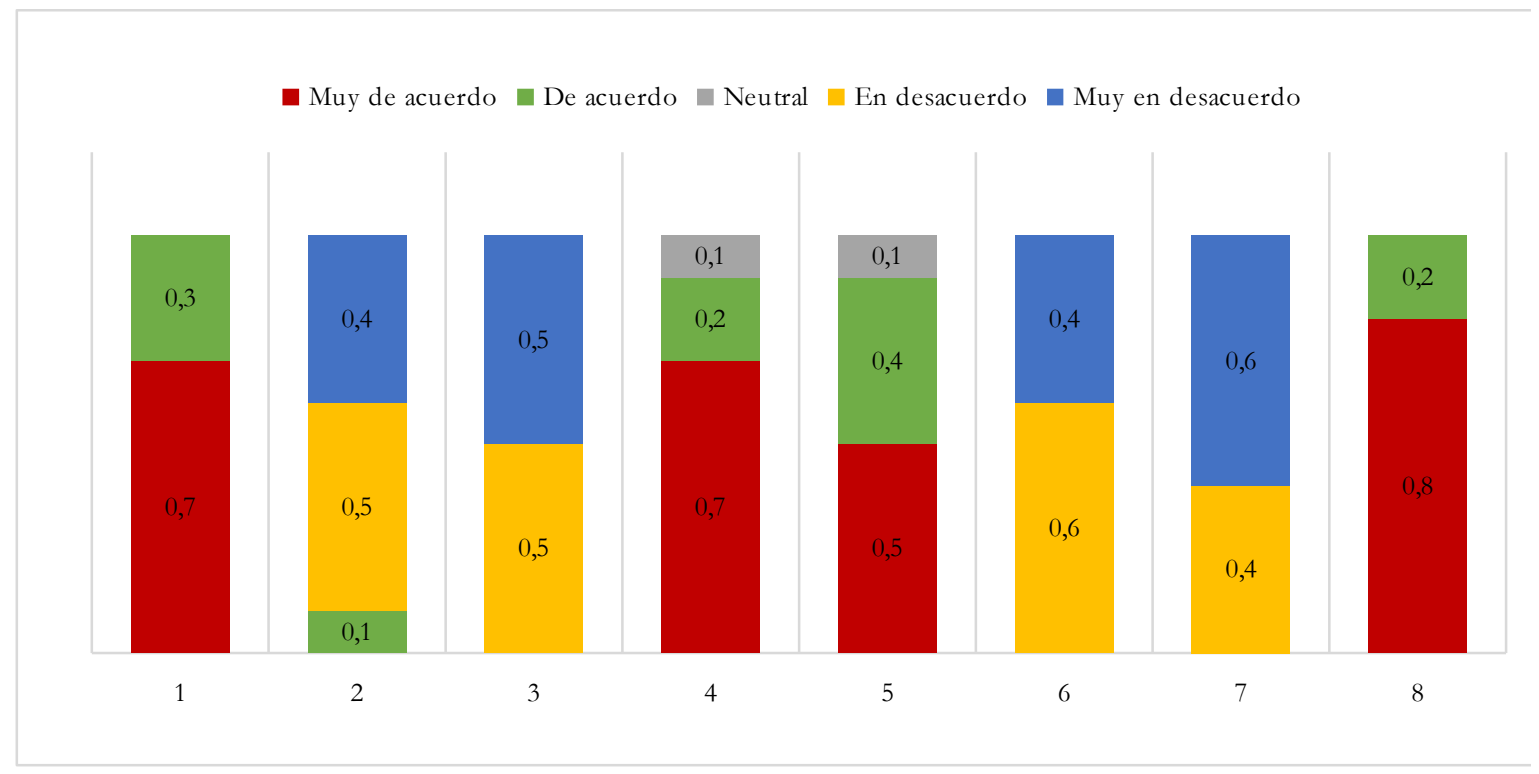

Figura 7. Resultados de la encuesta de satisfacción realizada entre los estudiantes respecto a la metodología empleada durante el curso. (1) La estrategia usada en esta asignatura es un estilo útil de enseñanza y aprendizaje. (2) Yo habría aprendido mejor el contenido con una estrategia más tradicional que la que se usó en esta asignatura. (3) La estrategia usada en esta asignatura es inapropiada para clases universitarias. (4) La estrategia usada en esta asignatura me ayudó a aprender su contenido. (5) Asignaturas de otros departamentos deberían usar la estrategia de éste. (6) La estrategia usada en esta asignatura no es para mí. (7) La estrategia usada en esta asignatura perjudicó el desarrollo de mi creatividad. (8) La estrategia usada en esta asignatura permitió una mejor aplicación de los contenidos.

\section{Conclusiones}

La creación del juego de mesa y su aplicación en el aula como resultado de un proyecto grupal del curso ha resultado ser una magnífica herramienta puntual en la enseñanza de la geomorfología que facilita la formación de geólogos en divulgación. Los estudiantes manifiestan una alta aceptación de la metodología de ABP empleada, siendo los protagonistas del aprendizaje en todo momento, lo que les permite reconocer el valor que tiene su participación como individuo y no tan solo la valoración numérica en sus notas en la de adquisición de conocimiento teórico. Además, se percibe un mayor nivel de interés y motivación que fomentan habilidades como la creatividad, el pensamiento crítico y conciencia social, ensalzando su rol fundamental como divulgadores de la geología.

Durante el desarrollo de la creación del juego y finalmente su empleo como tal, las clases teóricas se convirtieron en clases dinámicas, donde los estudiantes se involucraron para 
comprender la génesis de las formas y dominios geomorfológicos y se establecieron niveles competitivos entre ellos, motivando la mejora de la comprensión de formas complejas. Además, a través de la vinculación de la geomorfología con los diferentes pueblos nativos, los estudiantes fomentaron los sentimientos de interacción social y cultural y arraigo con la historia de los pueblos chilenos, esfuerzo que la implementación de la gamificación permitió.

El uso de APB en las aulas no sustituye la presencia ni la actividad del profesor, ya que el profesor sigue siendo pieza clave como guía en la innovación y el desarrollo del proyecto de gamificación. Es por tanto importante que el diseño de las actividades sea adecuado y se realice un seguimiento y evaluación continuados.

El potencial de investigar e innovar en modelos y estructuras de enseñanza endo-exo universitaria puede crear un impacto muy importante y positivo a corto plazo, tanto para las carreras de geología como para la sociedad, así como posibilidades de colaboración más directa entre los alumnos y agentes importantes de la educación fuera del ámbito universitario.

El juego creado es una magnífica alternativa para enseñar y divulgar el patrimonio geológico chileno de una manera diferente, haciendo más fácil el acceso a toda la sociedad. En contraste a las barreras económicas para la implementación de la gamificación que señala Mumtaz (2000), el coste económico que supuso la creación de este juego fue muy pequeño (menos de 20 USD), por lo que las oportunidades que ofrece este tipo de juego para la divulgación de la geología de Chile pueden ser inmensas. Por otra parte, las TIC han respaldado el crecimiento y la diversificación de la oferta y la calidad académica a la vez que reducen las diferencias sociales (Aylwin, Farcas y Yawan 2007), por lo que se propone una mayor socialización y accesibilidad al juego con la creación de una aplicación móvil o juego virtual. Además, realizando las modificaciones adecuadas, el juego se puede adaptar a otras regiones del planeta, manteniendo la mecánica del mismo.

Aunque a corto plazo los resultados de la de ABP en la enseñanza universitaria de la geología han sido satisfactorios, tienen que ser evaluados en mayor profundidad y a largo plazo. Las encuestas de percepción deben ser validadas con entrevistas personales y resulta necesario optimizar e investigar la adquisición de las habilidades y hábitos de análisis del medio natural y metodologías de divulgación para el desempeño de la carrera profesional en geología.

\section{Agradecimientos}

Este trabajo ha sido posible gracias al apoyo de Programa de Desarrollo Docente en Metodologías Activas de la Facultad de Ingeniería de la Universidad Andrés Bello. Los autores agradecen especialmente a Genaro Zavala y Ángeles Domínguez, de la Unidad de Desarrollo Docente y Académico (UNIDA), sus consejos y constante motivación durante el desarrollo de este trabajo.

\section{Referencias}

Arambarri J., Armentia L., Baeza U. (2012) Serious games para la puesta en valor de la cultura. Un caso práctico: SUM. Virtual Archaeology Review 3 (7), 65-67.

Arellano-Pimentel J. J., Nieva-García O., Algredo-Badillo I. (2013) Aprendizaje Basado en Proyectos Utilizando L-Systems en un Curso de Compiladores. Programación Matemática y Software 5 (1). pp. 82-96.

Aylwin M., Farcas D., Yawan L. (2007) New Technologies in Higher Education: Experiences from Chile and China. París: UNESCO.

Bell S. (2010) Project-Based Learning for the 21st Century: Skills for the Future. The Clearing House 83 (2), 39-43. 
Cascales A., Carrillo M. E., Redondo A. M. (2017) ABP y Tecnología en Educación Infantil. Píxel-Bit. Revista de Medios y Educación 50, 201-210.

Davis R. L. (2002) The value of teaching about geomorphology in non-traditional settings. Geomorphology 47 (2-4), 251-260.

De Bie M.H., Lipman L. J. A. (2012) The use of digital games and simulators in veterinary education: an overview with examples. Journal of Veterinary Medical Education 39 (1), 13 20 .

Faiella F., Ricciardi M. (2015) Gamification and learning: a review of issues and research. Journal of e-Learning and Knowledge Society 11 (3), 13-21.

Gaete Feres H. G. (2006) Chile: Los recursos del país al sistema de educación superior y su distribución regional. Osorno: Centro de Documentación Universitaria. Universidad de Los Lagos.

Gates A. E., Kalczynski M. J. (2016) The oil game: Generating enthusiasm for geosciences in urban youth in Newark, NJ. Journal of Geoscience Education 64 (1), 17-23.

Gómez B., Santos A. (2012) Competencias para la inserción laboral: Guía del profesorado. Madrid: Ministerio de Educación, Cultura y Deporte (España).

González F., Moreno C., Sáez R., Donaire T., Morales J. A., Toscano M., Fernández-Caliani J. C., Borrego J. (2012) Itinerarios geológicos virtuales: una experiencia docente desarrollada por alumnos. pp. 132-138 en Comunicaciones - XVII Simposio sobre Enseñanza de la Geología, Huelva.

González Tardon C. (2015) Comunicación corporativa gamificada en la universidad. Gamificación en redes sociales, experiencias, oportunidades y desventajas. communication papers. Media Literacy and Gender Studies 4 (8), 11-20.

Hamari J., Koivisto J., Sarsa H. (2014) Does gamification work? - A literature review of empirical studies on gamification. pp. 3025-3034 en System Sciences (HICSS), 47th Hawaii International Conference on System Sciences. Hawaii.

Hanus M. D., Fox J. (2015) Assessing the effects of gamification in the classroom: A longitudinal study on intrinsic motivation, social comparison, satisfaction, effort, and academic performance. Computers \& Education 80, 152-161.

Holubova R. (2008) Effective teaching methods - Project-based learning in physics. US-China Education Review 5 (12), 27-36.

Iosup A., Epema D. (2014) An experience report on using gamification in technical higher education. pp. 27-32 en Proceedings of the 45th ACM Technical Symposium on Computer Science Education. Atlanta (USA).

Kapp K. M. (2012) The gamification of learning and instruction: game-based methods and strategies for training and education. San Francisco: John Wiley \& Sons.

Kiovisto J., Hamari J. (2014) Demographic differences in perceived benefits from gamification. Computers in Human Behavior 35, 179-188.

Lim, K. Y. T. y Ong, M. Y. C. (2012). The Rise of Li' Ttledot: A study of citizenship education through game-based learning. Australasian Journal of Educational Technology 28 (8), 1420 1432.

Maldonado Pérez M. (2008) Aprendizaje basado en proyectos colaborativos. Una experiencia en educación superior. Laurus 14 (28), 158-180. 
Marín-Díaz V. (2015) La Gamificación educativa. Una alternativa para la enseñanza creativa. Digital Education Review 27.

Martí-Parreño J., Seguí-Mas D., Seguí-Mas E. (2016) Teachers' attitude towards and actual use of gamification. Procedia-Social and Behavioral Sciences 228, 682-688.

Mayer R. E. (2004) Should there be a three-strikes rule against pure discovery learning? American Psychologist 59(1), 14.

Morales J. A. (2012) El uso de Facebook como herramienta en la enseñanza de las Ciencias de la Tierra. XVII Simposio sobre Enseñanza de la Geología, Huelva. 275-278.

Mumtaz S. (2000) Factors affecting teachers' use of information and communications technology: a review of the literature. Journal of Information Technology for Teacher Education 9(3), 319-342.

Sánchez Martínez A., Rojas Molina A., Sánchez Flores L. (2015) Transformando la educación tradicional a través del aprendizaje basado en proyectos y el uso de las tecnologías de la información y comunicación. Repositorio Digital Universitario de Materiales Didácticos. Universidad Nacional Autónoma de México. pp.1-18.

Shipley T. F., Tikoff B., Ormand C., Manduca C. (2013) Structural geology practice and learning, from the perspective of cognitive science. Journal of Structural Geology 54, 72-84.

Smith D. L., Hoersch A. L., Gordon P. R. (1995) Problem-based learning in the undergraduate geology classroom. Journal of Geological Education 43(4), 385-390.

Tishman S., Jay E., Perkins D. N. (1993) Teaching thinking dispositions: From transmission to enculturation. Theory into Practice 32(3), 147-153.

Urh M., Vukovic G., Jereb E. (2015) The model for introduction of gamification into elearning in higher education. Procedia-Social and Behavioral Sciences 197, 388-397.

Williams M. S. (2014) PBL Field Deployment: Lessons Learned Adding a Problem-Based Learning Unit to a Traditional Engineering Lecture and Lab Course, 121st ASEE Annual Conference and Exposition, Indianapolis.

Yang J. C., Chien K. H., Liu T. C. (2012) A digital game-based learning system for energy education: An energy conservation pet. The Turkish Online Journal of Educational Technology 11(2), 27-37.

Zichermann G., Cunningham C. (2011) Gamification by design: Implementing game mechanics in web and mobile apps. Sebastopol (Canadá): O'Reilly Media. Inc. 